\title{
Comparison of Bag and Non-Bag Extraction of Gall Stones through Laparoscopy
}

\author{
IJCRR \\ Section: Healthcare \\ ISI Impact Factor \\ (2019-20): 1.628 \\ IC Value (2019): 90.81 \\ SJIF (2020) $=7.893$ \\ (c) (i) (3) \\ Copyright@IJCRR
}

\section{Adith Chinnaswami, Prabhu Purushothaman, Naresh Duthaluri, Rekha Arcot}

Sri Ramachandra lnstitute of Higher Education and Research, No. 1, Ramachandra Nagar, Porur, Chennai, India.

\section{ABSTRACT}

Introduction: Laparoscopic cholecystectomy (LC) has become the gold standard surgical procedure of choice for disorders involving the gall bladder and biliary tract. There are several intraoperative and postoperative complications associated with LC of which port site infections(PSI) are associated with high morbidity and mortality. The present study was carried out to compare the outcomes with bag and non -bag extraction of gall bladder in terms of the incidence of PSI.

Methods: This randomized controlled trial was carried out among 326 adults who underwent LC for two years. The participants were randomized into bag extraction and non-bag extraction groups. Postoperatively, the participants were followed up for one week to evaluate the incidence of PSI.

Results: The incidence of PSI among bag extraction was $1.4 \%$ compared to $9.1 \%$ in the non-bag extraction. The presence of diabetes mellitus, elevated glycosylated haemoglobin and immunocompromised status were proven to be risk factors for PSI. $(p<0.001)$

Conclusion: Since bag extraction is associated with lower rates of infection, it is advisable to follow bag extraction as a routine procedure in all LC surgeries, especially in high-risk groups like diabetes mellitus and immunocompromised states.

Key Words: Bag extraction, End glove, Gall bladder diseases, Laparoscopic Cholecystectomy, Port site infections, Randomized controlled trial

\section{INTRODUCTION}

From the time of establishing cholecystectomy as the most preferred treatment option for cholelithiasis, surgical advancements have been on the rise and, for the past few decades, laparoscopic cholecystectomy (LC) has become the gold standard surgical procedure of choice for the disorders involving gall bladder and biliary tract. The reliability with LC is significantly higher, due to lower incidences of complications ranging from $1 \%-6 \% .{ }^{1} \mathrm{LC}$ has, in recent times, replaced open cholecystectomy to a large extent except in cases of patients not being fit for general anaesthesia, or, in the presence of malignancies and intra-operative complications leading to conversion from laparoscopic procedure to an open procedure. The rates of such conversions have considerably reduced in the past two decades owing to an increase in the expertise of the surgeons, better understanding of the patient selection and improvement in laparoscopic instrumentation. According to studies published by Kaushik $\mathrm{R}$ et al. in a single-institution prospective study, the overall conversion rate was found to be $7.06 \% .^{2}$

The complications of LC, all though few in numbers, continue to challenge the surgeons about morbidity and mortality. The intraoperative complications can arise at any point during the surgery, from induction of the patient under general anaesthesia, injury during trocar insertion, respiratory compromise during carbon dioxide insufflation, injury to adjacent structures during dissection, common bile duct injury, trauma to the liver during dissection of the gall bladder, perforation of gall bladder leading to bile leak and spillage of stones during retrieval of the gall bladder. Post-operative complications are usually secondary to intraoperative complications, such as bile leak leading to biliary peritonitis or biliary fistula, spilt stones causing abscess formation. Port site complications such as port-site infections(PSI), port-site hernias and port site metastases have also been reported. According to a review done by

\section{Corresponding Author:}

Dr. Prabhu Purushothaman, Sri Ramachandra Institute of Higher Education and Research, No.1, Ramachandra Nagar, Porur, Chennai, India. Phone: 7736124616; Email: dr.jpprabhu@gmail.com
ISSN: 2231-2196 (Print)
ISSN: 0975-5241 (Online)

Received: 04.02 .2021

Revised: 26.03 .2021

Accepted: 12.05 .2021

Published: 24.10 .2021 
Sasmal PK et al., the umbilical PSI is far more common than many other complications, ranging from $8 \%$ to $89 \%{ }^{3}$

Though the complications associated with laparoscopic cholecystectomy have decreased significantly over the past decade, in the quest to provide better patient care and further reduce chances of complications, it is important to be aware of the possible risk factors associated with various complications and how to reduce the chances of any complication associated with the surgery. With this background, the present study was undertaken to compare the post-operative port site wound infections in laparoscopic cholecystectomy between extraction of gall bladder by bag extraction versus non-bag extraction, and also evaluate the risk of PSI with various factors such as diabetes mellitus, Body mass index, hypoalbuminemia and the immuno-compromised status of patients.

\section{METHODOLOGY}

\section{Study setting and participants}

This randomized controlled study was carried out in the Department of General Surgery of our tertiary teaching institution among all the adult patients (aged $>18$ years) undergoing laparoscopic cholecystectomy (LC) for two years between January 2018 and December 2019. Patients with empyema of the gall bladder and those who were intraoperatively converted to open cholecystectomy were excluded from the analysis. A total of 326 participants were taken up for the study.

\section{Randomization and blinding}

The participants were randomized into either bag extraction or non-bag extraction of gall bladder before surgery. This was done by the sealed envelope technique. The study was carried out as a single-blind study.

\section{Procedure}

After obtaining informed consent, patients underwent routine preoperative workup and anaesthetic assessment. After adequate optimization, in a controlled setting, patients were taken up for surgery. Extraction of the gall bladder was done through the epigastric port. In non-bag extraction, the gall bladder was directly grasped with a claw and retrieved via the epigastric port. In the bag extraction group, a sterile plastic endo bag was created by cutting a sterilized bag and putting a purse-string suture around the mouth with a Roeder's knot to tighten it. This bag was introduced through the $10 \mathrm{~mm}$ port after the resection of the gall bladder from the gall bladder fossa. The gall bladder was manoeuvred into the end bag and mouth closed by tightening the Roeder's knot. The specimen was then extracted through the epigastric port. Standard antibiotic protocol of three doses of Ceftriaxone, a third-generation cephalosporine dose pre-operatively and two doses postoperatively was followed uniformly for all the study participants.

Postoperatively, the port site used for gall bladder extraction was monitored for seven days to check for wound site infection. In case of any signs of an infection (pain, erythema, swelling or discharge), a wound swab was taken and sent for culture. The presence of positive growth on the wound culture was considered as port site infection. Participants were subsequently started on antibiotics for further management.

\section{Data collection}

A structured proforma was used to record demographic and other clinical particulars including diabetes mellitus and immunocompromised status of the study participants. Body mass index was measured and documented. The laboratory parameters including glycosylated haemoglobin, serum albumin and serum creatinine were documented. The underlying pathology of the gall bladder or biliary tract was also documented as infective or non-infective.

\section{Data analysis}

Data was entered and analyzed using SPSS ver 20 software. The incidence of port-site infections was documented as percentages. The comparison between bag and non-bag extraction was carried out using the chi-square test. A p-value $<0.05$ was considered statistically significant.

\section{RESULTS}

Out of the 326 participants that underwent laparoscopic cholecystectomy, $58 \%$ of them were female and $42 \%$ of the patients were male. In the bag extraction group, $57.1 \%$ were female and $42.9 \%$ male. In this study $31.6 \%$ of the patients were diabetic and $26.4 \%$ had an infective pathology In the bag extraction group, $22.9 \%$ had infective pathology whereas $77.1 \%$ had non-infective pathology. (Table 1)

In the bag extraction group, the mean $\mathrm{HbA1C}$ level was 5.9 whereas the same in the non-bag extraction group was 5.6. The bag extraction group showed a mean body mass index (BMI) of $26.24(\mathrm{SD} \pm 3.2)$ while the non-bag extraction group showed a mean of 24.9 ( $\mathrm{SD} \pm 4$ ). The mean serum creatinine for the 140 patients in the bag extraction group was 1.1 and the same in the non-bag extraction group was 1.2. (Table 2)

In this study, the overall infection rate among the study participants was $5.82 \%$. Further analysis shows an infection rate of $1.4 \%$ in the bag extraction group and $9.1 \%$ in the non-bag extraction group. The observed difference was statistically significant $(\mathrm{p}<0.05)$ (Table 3$)$

In non-bag extraction of the gall bladder, 17 out of the 186 patients in this group developed postoperative port-site in- 
fections. This showed a $9.1 \%$ infective rate as compared to $1.4 \%$ in the bag extraction rate. On multi-variate analysis of the parameters, in the patients who developed infections in the non-bag extraction group, presence of diabetes, elevated $\mathrm{HbA1C}$, immunocompromised status and infective pathology were found to have statistically significant differences in the outcomes $(p<0.05)$. (Table 4$)$

\section{DISCUSSION}

Minimal access surgery, especially laparoscopic surgery is now the procedure of choice for gall bladder related pathologies due to its advantage of a smaller incision, shorter recovery time and decreased post-operative pain. The common postoperative complications in laparoscopic cholecystectomy include port site hernias, port-site metastases and port site infections. ${ }^{4}$ However, there has been a steady increase in the incidence of infectious complications post laparoscopic cholecystectomy. ${ }^{5}$ These infections can be attributed to complications such as unretrieved stones and bile spillage. ${ }^{5}$ In addition, lost gall stones are a significant source of postoperative morbidity. ${ }^{6,7}$ Slipping of the cystic duct clip or tearing of the gall bladder during retrieval can result in spillage of stones. ${ }^{8,9}$ Perforation of the gall bladder either during the surgery or during specimen retrieval leads to the spillage of bile. In patients with acute cholecystitis, the gall bladder is friable, thus making it susceptible to tears. In addition, the presence of adhesions also makes dissection more difficult, leading to an increased risk of perforation. ${ }^{5}$ These complications can be avoided by the use of an endo bag for retrieval of the gall bladder. The conventional method for the removal of the gall bladder is associated with a higher incidence of infection as reflected in the study conducted by Naeem Taj et al., in which $5.28 \%$ of patients who underwent extraction without endoglove developed an infection as opposed to $0.20 \%$ of patients in whom gall bladder was extracted with an endoglove. ${ }^{5}$ These results concur with the present study in which $9.1 \%$ of patients developed infections with non-bag extraction and $1.4 \%$ developed infection with endobag extraction.

The overall mean age of the patients in the study was 48.27( $\mathrm{SD} \pm 14.606)$. A study conducted by Saud JD et al. showed that the male gender has a slightly increased risk of developing postoperative surgical site infections. ${ }^{10}$ In this study, $58 \%$ were females and $42 \%$ of males. Studies conducted by Taj MN et al. showed that diabetic patients were at a higher risk of developing post-operative infections. ${ }^{5}$.In this study, the overall rate of diabetes amongst patients who underwent laparoscopic cholecystectomy was 31.6\%. On analyzing the patients who developed infections in the nonbag extraction group, $52.9 \%$ of the patients were found to be diabetic $(p<0.001)$. Similar observations were seen with glycosylated haemoglobin $(\mathrm{p}<0.0005)$. This is similar to the results reported in the study conducted by Taj MN et al. where $44 \%$ of the patients who developed port site infections were diabetic. ${ }^{5}$ This suggests that bag extraction would be beneficial to patients who are diabetic, by reducing the risk of port-site infections.

In the study done by Taj MN et al., the frequency of infections was more in the patients with infective pathology. ${ }^{5}$ Infective pathology including empyema of gall bladder and acute cholecystitis accounted for $70 \%$ of the patients who developed port site infections. ${ }^{5}$ In this study, patients with an infective pathology constituted $82.4 \%$ of the patients who developed port site infections $(\mathrm{p}<0.0005)$. This suggests that bagging must be routinely used in cases with an infective pathology to avoid port site infections. In this study, $10.1 \%$ of the entire population which underwent laparoscopic cholecystectomies were immuno- compromised. On analyzing the patients who developed port-site infections in the non-bag extraction group, $41.2 \%$ were found to be immuno-compromised $(p<0.002)$. It may be preferable to carry out bagging as a routine procedure in patients with immunocompromised status to prevent port site infections.

\section{CONCLUSION}

An infection rate of $1.4 \%$ on bag extraction as opposed to $9.1 \%$ on non-bag extraction shows that the incidence of portsite infections can be reduced by the use of a sterile endobag for the extraction of gall bladder following laparoscopic cholecystectomy.

While bagging may be considered cumbersome or may marginally increase the operating time, selective bagging must be practised. Diabetes elevated glycosylated haemoglobin levels were also found to be significant factors in causing port site infections. Thus, bag extraction must be routinely followed in diabetic patients, especially those with high glycosylated haemoglobin levels. Bagging may be routinely followed in patients with retro-positive status, exposure to tuberculosis, having undergone chemotherapy or radiotherapy or having a history of steroid intake.

\section{ACKNOWLEDGEMENT}

The authors acknowledge the immense help received from the scholars whose articles are cited and included in references of this manuscript. The authors are also grateful to authors/editors/publishers of all those articles, journals and books from where the literature for this article has been reviewed and discussed.

\section{Declaration}

\section{Conflict of interest: nil}




\section{Source of Funding: nil}

Ethical approval obtained: (REF: CSP-MED/15/OCT/25/51)

\section{REFERENCES}

1. Singh K, Ohri A. Difficult laparoscopic cholecystectomy: A Difficult laparoscopic cholecystectomy: A large series from north India. Indian J Surg. 2006; 68: 205-08.

2. Kaushik R, Sharma R, Batra R, Yadav TD, Attri AK, Kaushik SP et al. Laparoscopic Cholecystectomy: An Indian Experience of 1233 Cases. J Laparoendosc Adv Surg Tech A. 2002;12(1):21-5.

3. Sasmal PK, Mishra TS, Rath S, Meher S, Mohapatra D. Port site infection in laparoscopic surgery: A review of its management. World J Clin Cases. 2015; 3(10): 864-871.

4. Mir IS. Minimal access surgery port-site complications. JK Science. $2003 ; 10(3): 226-8$
5. Taj MN, Naeem M, Iqbal Y, Akbar Z. Frequency and prevention of laparoscopic port site infection. J Ayub Med Coll Abbottabad.2012; 24: 197-199.

6. Brockmann JG, Kocher T, Senninger NJ, Schurmann GM. Complications due to gall stones lost during LaparosocpicCholecystectomy: An analysis of incidence, clinical course and management. Surg Endosc. 2002;16: 1226 -32

7. SatheshKumar T, Saklani AP, Vinayagam R, Blackett RL. Spilt gallstones during laparoscopic cholecystectomy: a review of the literature. Postcard Med J 2004; 80: 77-9

8. Läuffer JM, Krahenbuhl L, Baer HU, Mettler M, Buchler MW. Clinical manifestations of lost gallstones after laparoscopic cholecystectomy: a case report with review of the literature. Surg Laparosc Endosc 1997;7:103 -12.

9. Hackan DJ, Rotstein OD. Host response to laparoscopic surgery: mechanisms and clinical correlates. Can J Surg 1998;41:103-11

10. Saud JD, Abu Al-Hail MC. Surgical site infections after laparoscopic cholecystectomy. Bas J Surg 2010:1-6.

Table 1: Background characteristics of the study participants:

\begin{tabular}{|c|c|c|c|c|c|c|}
\hline \multirow[t]{2}{*}{ S. No } & \multirow[t]{2}{*}{ Characteristics } & & \multicolumn{2}{|c|}{ Bag extraction } & \multicolumn{2}{|c|}{ Non-bag extraction } \\
\hline & & & $\mathbf{N}$ & $\%$ & $\mathbf{N}$ & $\%$ \\
\hline \multirow[t]{2}{*}{1} & \multirow[t]{2}{*}{ Gender } & Female & 80 & 57.1 & 109 & 58.6 \\
\hline & & Male & 60 & 42.9 & 77 & 41.4 \\
\hline \multirow[t]{2}{*}{2} & \multirow[t]{2}{*}{ Diabetes } & No & 94 & 67.1 & 129 & $69 \cdot 4$ \\
\hline & & Yes & 46 & 32.9 & 57 & 30.6 \\
\hline \multirow[t]{2}{*}{3} & \multirow[t]{2}{*}{ Infective pathology } & Infective pathology & 32 & 22.9 & 54 & 29 \\
\hline & & Non-infective Pathology & 108 & 77.1 & 132 & 71 \\
\hline \multirow[t]{2}{*}{4} & \multirow{2}{*}{$\begin{array}{l}\text { Immunocompromised } \\
\text { status }\end{array}$} & No & 122 & 87.1 & 171 & 91.9 \\
\hline & & Yes & 18 & 12.9 & 15 & 8.1 \\
\hline
\end{tabular}

Table 2: Laboratory parameters:

\begin{tabular}{llcccc} 
S. No & Characteristics & Bag extraction & \multicolumn{3}{c}{ Non-bag extraction } \\
& & Mean & SD & Mean & SD \\
\hline 1 & Glycosylated hemoglobin $(\mathrm{HbAlC})$ & 5.9 & 1.4 & 5.6 & 1.4 \\
2 & Body mass index & 26.2 & 3.2 & 24.9 & 4. \\
3 & Serum albumin & 3.6 & 0.9 & 3.8 & 0.7 \\
4 & Serum creatinine & 1.1 & 0.5 & 1.2 & 0.5 \\
\hline
\end{tabular}

Table 3: Incidence of infections:

\begin{tabular}{|c|c|c|c|c|c|c|c|}
\hline \multirow[t]{2}{*}{ S. No. } & \multirow[t]{2}{*}{ Characteristics } & \multicolumn{2}{|c|}{ Infection } & \multicolumn{2}{|c|}{ No infection } & \multirow[t]{2}{*}{ chi sq } & \multirow[t]{2}{*}{ P-value } \\
\hline & & $\mathbf{N}$ & $\%$ & $\mathbf{N}$ & $\%$ & & \\
\hline 1 & Bag extraction & 2 & 1.4 & 138 & 98.6 & \multirow{2}{*}{8.65} & \multirow{2}{*}{$<0.05$} \\
\hline 2 & Non bag extraction & 17 & 9.1 & 169 & 90.9 & & \\
\hline
\end{tabular}

Table 4: Factors influencing the risk of infections:

$\begin{array}{llcc}\text { S. No } & \text { Characteristics } & \text { P-value with bag extraction } & \text { P-value with non-bag extraction } \\ 1 & \text { Age } & 0.980 & 0.577 \\ 2 & \text { Sex } & 0.131 & 0.940 \\ 3 & \text { Diabetes } & 0.114 & 0.001^{*}\end{array}$


Table 4: (Continued)

\begin{tabular}{llcc} 
S. No & Characteristics & P-value with bag extraction & P-value with non-bag extraction \\
4 & Glycosalated Hb & 0.730 & $0.0005^{*}$ \\
5 & Body Mass Index & 0.498 & 0.462 \\
6 & Serum Albumin & 0.142 & 0.732 \\
7 & Serum Creatinine & 0.975 & 0.314 \\
8 & Immuno-Compromised & 0.161 & $0.002^{*}$ \\
& status & & \\
9 & Infective pathology & 0.418 & $0.0005^{*}$ \\
\hline
\end{tabular}

*highly significant 